\title{
BMJ Open Which patients benefit specifically from short-term psychodynamic psychotherapy (STPP) for depression? Study protocol of a systematic review and meta-analysis of individual participant data
}

\author{
Ellen Driessen, ${ }^{1}$ Allan A Abbass, ${ }^{2}$ Jacques P Barber, ${ }^{3}$ \\ Mary Beth Connolly Gibbons, ${ }^{4}$ Jack J M Dekker, ${ }^{5}$ Marjolein Fokkema, ${ }^{6}$ \\ Peter Fonagy, ${ }^{7}$ Steven D Hollon, ${ }^{8}$ Elise P Jansma, ${ }^{9}$ Saskia C M de Maat, ${ }^{10}$ \\ Joel M Town, ${ }^{2}$ Jos W R Twisk, ${ }^{11,12}$ Henricus L Van, ${ }^{10}$ Erica Weitz, ${ }^{1}$ Pim Cuijpers ${ }^{1}$
}

To cite: Driessen E, Abbass AA, Barber JP, et al. Which patients benefit specifically from short-term psychodynamic psychotherapy (STPP) for depression? Study protocol of a systematic review and meta-analysis of individual participant data. BMJ Open 2018;8:e18900. doi:10.1136/ bmjopen-2017-018900

\section{- Prepublication history for} this paper is available online. To view these files, please visit the journal online (http://dx.doi. org/10.1136/bmjopen-2017018900).

Received 28 July 2017 Revised 16 October 2017 Accepted 21 December 2017

Check for updates

For numbered affiliations see end of article.

Correspondence to

Dr Ellen Driessen;

e.driessen@vu.nl

\section{ABSTRACT}

Introduction Short-term psychodynamic psychotherapy (STPP) is an empirically supported treatment that is often used to treat depression. However, it is largely unclear if certain subgroups of depressed patients can benefit specifically from this treatment method. We describe the protocol for a systematic review and meta-analysis of individual participant data (IPD) aimed at identifying predictors and moderators of STPP for depression efficacy. Method and analysis We will conduct a systematic literature search in multiple bibliographic databases (PubMed, PsycINFO, Embase.com, Web of Science and Cochrane's Central Register of Controlled Trials), 'grey literature' databases (GLIN and UMI ProQuest) and a prospective trial register (http://www.controlled-trials. com). We will include studies reporting (a) outcomes on standardised measures of (b) depressed (c) adult patients (d) receiving STPP. We will next invite the authors of these studies to share the participant-level data of their trials and combine these data to conduct IPD meta-analyses. The primary outcome for this study is post-treatment efficacy as assessed by a continuous depression measure. Potential predictors and moderators include all sociodemographic variables, clinical variables and psychological patient characteristics that are measured before the start of treatment and are assessed consistently across studies. One-stage IPD meta-analyses will be conducted using mixed-effects models.

Ethics and dissemination Institutional review board approval is not required for this study. We intend to submit reports of the outcomes of this study for publication to international peer-reviewed journals in the fields of psychiatry or clinical psychology. We also intend to present the outcomes at international scientific conferences aimed at psychotherapy researchers and clinicians. The findings of this study can have important clinical implications, as they can inform expectations of STPP efficacy for individual patients, and help to make an informed choice concerning the best treatment option for a given patient.
Strengths and limitations of this study

- This is the first study that systematically assesses patient characteristics associated with shortterm psychodynamic psychotherapy (STPP) for depression efficacy.

- Individual participant data (IPD) meta-analysis allows for the examination of predictors and moderators by maximising statistical power while protecting against ecological bias that presents problems when using conventional meta-analysis techniques.

- The findings of this study can have important clinical implications, as they can inform expectations of STPP efficacy for individual patients, and help to make an informed choice concerning the best treatment option for a given patient.

- IPD meta-analyses rely on variables previously assessed in individual studies and available across multiple trials. Thus, it is possible that not all variables of interest can be examined as potential predictors or moderators.

PROSPERO registration number CRD42017056029.

\section{INTRODUCTION}

Depression is a highly prevalent and disabling disorder associated with major personal and societal costs. ${ }^{1}$ Affecting more than 300 million people worldwide, depression is ranked as the single largest contributor to global disability by the WHO. ${ }^{2}$ Given the tremendous burden of disease, there is a great need for effective and efficient treatments for depression. Antidepressant medications and different psychological therapies constitute 
the predominant treatments for depressive disorders. ${ }^{3}$ Concerning psychological treatments, there is a clinical tradition of short-term psychodynamic psychotherapies (STPPs) being used to treat depression. STPP is an empirically supported treatment for depression. ${ }^{4}$ However, it is unlikely that any treatment will work equally well for all depressed patients, ${ }^{5}$ and it remains largely unclear if certain subgroups of patients can benefit specifically from STPP.

Two types of information are relevant to this question: predictors and moderators. Predictors (or prognostic factors) predict outcome to a given treatment and can be used to determine which patients are more likely to respond to STPP relative to other patients. For instance, if age were found to be a positive predictor of STPP efficacy, this might indicate that older patients would be more likely to benefit from STPP than younger patients. Predictors can inform expectations of STPP efficacy, but are of little use in deciding which treatment to select. On the other hand, moderators (or prescriptive factors) can detect different patterns of outcomes between different treatments for different types of patients and provide a basis for choosing the best treatment for a given patient. ${ }^{6}$ For instance, if age were found to be a moderator of STPP efficacy versus antidepressant medication, this might indicate that older patients might benefit more from STPP than from medication, while younger patients might benefit more from medication than from STPP.

Some preliminary empirical findings concerning predictors and moderators of STPP efficacy for depression do exist. With regard to predictors, meta-regression analyses alongside a 'conventional' meta-analysis (based on results extracted from publications ${ }^{4}$ ) showed that mean pretreatment depression scores were positively associated with pretreatment to post-treatment depression effect size, although this effect might not be specific to STPP. ${ }^{7}$ With regard to moderators, Driessen et $a l^{8}$ found STPP to be more efficacious than CBT among depressed patients who showed low baseline comorbid anxiety levels. Furthermore, Barber et al ${ }^{9}$ reported that STPP was more efficacious than medication or placebo for ethnic minority males. However, a systematic assessment of which patient characteristics are associated with STPP efficacy is currently lacking.

The main reason why predictors and moderators of STPP for depression have not yet been examined thoroughly is lack of statistical power in individual clinical trials due to relatively small sample sizes. Prediction and moderation analyses can also be conducted alongside conventional meta-analyses. However, since these analyses are usually based on study-level characteristics, they are prone to ecological bias, such that the association between the study-level characteristics may not be representative of the true relationships in the data at the individual level. ${ }^{10}$ Thus, current research methods (clinical trials and conventional meta-analyses) have been insufficient to answer the question as to whether certain subgroups of patients can benefit specifically from STPP for depression.

Individual participant data (IPD) meta-analysis is a relatively new technique to examine treatment effects by combining participant-level data of multiple trials. IPD meta-analysis uses the same basic approach as any other well-conducted systematic review and meta-analysis. However, it involves collection of the original data from as many of the relevant trials worldwide as can be accessed. IPD meta-analysis has several advantages over conventional meta-analysis, including increased statistical power to examine predictors and moderators of treatment efficacy. ${ }^{11}$ Furthermore, because predictors and moderators are studied at patient level, ecological bias can be circumvented. For these reasons, IPD meta-analysis is currently considered the 'gold standard' in evidence synthesis. ${ }^{12}$

We describe the study protocol for a systematic review and IPD meta-analysis concerning predictors and moderators of STPP for depression efficacy. The aim of this project is to examine which depressed patients benefit specifically from STPP in terms of depressive symptom reduction when compared with other patients (predictors), and which patients benefit specifically from STPP when compared with no-treatment conditions, other psychotherapies and antidepressant medication (moderators). The goal of this study is to collect the participant-level data of trials examining the efficacy of STPP for depression identified by a systematic literature search, and to combine these datasets in order to conduct IPD meta-analyses.

\section{METHODS \\ Design}

This study is a systematic review and meta-analysis of IPD that is registered in the PROSPERO International prospective register of systematic reviews (registration number: CRD42017056029). Important protocol amendments will be documented in this register too. The project started on 1 December 2016 and is expected to be completed by 31 July 2019 .

\section{Search strategy}

We will use an extensive search strategy including six different search methods in order to retrieve as many relevant studies as possible. These searches have already been performed in 2007 and 2014 for two previous conventional meta-analyses concerning the efficacy of STPP for depression ${ }^{413}$ and will be updated in 2017.

First, we will systematically search the bibliographic databases PubMed, PsycINFO (via EBSCO), Embase.com, Web of Science (via Elsevier) and Cochrane's Central Register of Controlled Trials (via Wiley). Search terms will include a wide range of synonyms, both in index terms and free-text words, for (1) psychodynamic psychotherapy (eg, psychotherapy, psychoanalytic), (2) therapy (eg, psychotherapy), (3) psychodynamic (eg, dynamic*) and (4) depression (eg, depressive disorder). These four 


\begin{tabular}{|c|c|c|}
\hline Search & PubMed query 19-06-2017 & Items found \\
\hline$\# 1$ & $\begin{array}{l}\text { Search "Psychoanalytic Therapy"[Mesh] OR "Psychotherapy, Psychodynamic"[Mesh] OR } \\
\text { psychodynamic*[tiab] Sort by: Relevance }\end{array}$ & 20177 \\
\hline \#2 & $\begin{array}{l}\text { Search ("Psychotherapy"[Mesh:noexp] OR “Animal Assisted Therapy"[Mesh] OR “Art } \\
\text { Therapy'(Mesh) OR “Bibliotherapy"[Mesh] OR “Psychotherapy, Group”[Mesh] OR "Psychotherapy, } \\
\text { Brief”[Mesh] OR "Psychotherapy, Multiple”[Mesh] OR “Counselling”[Mesh:NoExp] OR "Directive } \\
\text { Counselling"[Mesh:NoExp] OR ((psychotherap*[tiab] OR therap*[tiab] OR counselling[tiab]) NOT } \\
\text { medline[sb])) }\end{array}$ & 380901 \\
\hline \#3 & $\begin{array}{l}\text { Search dynamic*[tiab] OR STPP[tiab] OR BDT(tiab] OR DIT[tiab] OR insight*[tiab] OR interpretive[tiab] } \\
\text { OR interpretative[tiab] OR analytic*[tiab] OR psychoanalytic*[tiab] }\end{array}$ & 1073217 \\
\hline \#4 & Search \#2 AND \#3 & 21435 \\
\hline \#5 & Search \#1 OR \#4 & 39841 \\
\hline \#8 & 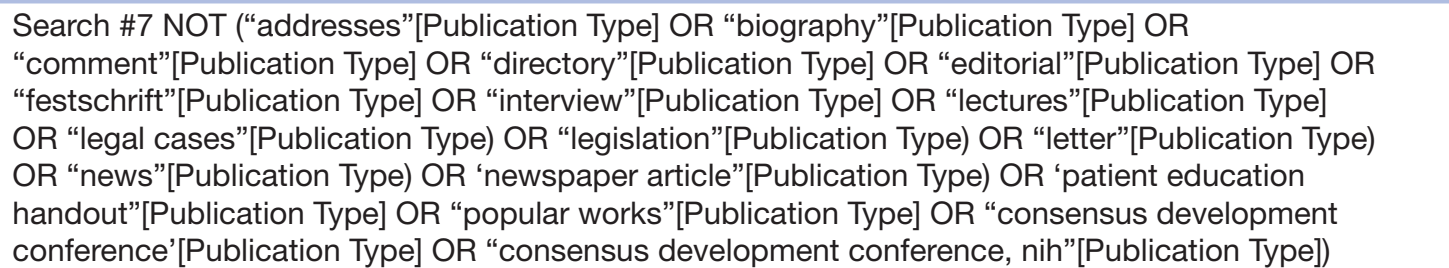 & 2285 \\
\hline
\end{tabular}

sets of search terms will be combined as follows: (\#1 OR (\#2 AND \#3) ) AND \#4. The exact terms for the search in PubMed are presented in table 1. Complete search terms for all electronic databases are available on request from the corresponding author. No language or date restrictions will be applied in the searches.

Second, in order to identify relevant studies from the so-called 'grey literature', we will search GLIN, a Dutch electronic database for grey literature, and UMI database ProQuest for digital dissertations. Third, a prospective trial register will be searched for unpublished ongoing research (http://www.controlled-trials.com). The grey literature and prospective trial register searches will be conducted using the search strategy described above. Fourth, we will search an Internet database of controlled and comparative outcome studies on psychological treatments of depression (http://www.psychotherapyrcts. $\mathrm{org}^{14}$ ) for studies examining STPP. Fifth, reviews and meta-analyses concerning the efficacy of psychodynamic treatments for depression or for psychiatric disorders in general retrieved from the first search method will be screened for relevant references not located by means of the other search methods. Sixth, we will contact an email list of researchers in the field of psychodynamic therapy to ask for ongoing or unpublished studies.

\section{Selection of studies}

We will include studies if they report (a) outcomes on standardised measures of (b) depressed (c) adult patients (d) receiving STPP. Participants are considered depressed if they meet specified criteria for major depressive disorder or another unipolar mood disorder as assessed by means of a semistructured interview or clinicians' assessment, or if they present an elevated score above the "no depression' cut-off on a standardised measure of depression. Participants need to be at least 18 years old, and studies concerning older adults (mean age $>55$ ) will be included as well. We will include studies in which STPP (a) is based on psychoanalytic theories and practices, (b) is time-limited from the onset (ie, not a therapy that is brief only in retrospect) and (c) applies verbal techniques (eg, therapies applying art as expression form are not considered STPP). Studies need to include at least 10 subjects. Case studies will therefore be excluded. We will also include naturalistic studies with a heterogeneous study sample, if these studies include more than 10 participants diagnosed as depressed, as these subgroups also meet the inclusion criteria specified previously. For these studies, the authors will be contacted with a request for subgroup data.

The screening process will consist of three phases. At first, the selection criteria will be applied to the citations generated from the searches independently by two raters. Disagreements will be discussed and resolved by consensus. Unless they can be definitely excluded, titles identified as potentially relevant will be requested in full text. During the second screening phase, two independent raters will apply the selection criteria to the full-text papers. Disagreements will be discussed and resolved by consensus. During the third phase, two expert STPP raters will confirm that the included papers meet criteria for STPP. Again, disagreements will be discussed and resolved by consensus. When disagreements cannot be resolved in this way, a third rater will be consulted. 


\section{Data collection}

Authors of the included studies will be contacted and invited to contribute the participant-level data of their studies. Researchers who share their data will be offered coauthorship for all publications that are based on their study's data (group-authorship), given that they meet standard criteria for authorship of scientific publications according to internationally accepted criteria (www. icmje.org). In addition, the collected data will be made available to investigators who contribute data to examine other research questions in the combined dataset. This strategy has been used in previous IPD meta-analyses concerning depression treatments ${ }^{15}$ and has been successful in convincing researchers to share their data.

Contact details of all first authors will be collected from the relevant publications, or if not reported there, through Internet searches or personal contacts with other researchers. First authors will be contacted by email with a letter of invitation outlining the project's goals and asking if they would be willing to collaborate by sharing the participant-level data of their trial. If an author does not respond after 3 weeks, a second and third email will be sent. In case of non-response to email, a letter will be sent (again with three attempts). If still no response is received, we will try to contact the author by telephone. If all these attempts fail, the last, second, third, fourth, etc. author of the study (in this order) will be contacted in the same way. If none of the authors respond to these efforts, other ways will be sought to contact one of the authors (eg, via colleagues or anyone who might know them). Study data will be considered unavailable only if all these attempts fail, or in the event that an author indicates that the participant-level data have not been retained or declines sharing these data.

If the author is willing and able to share the IPD of his/her trial, both parties will sign a data-sharing agreement. The author will then transfer the participant-level dataset, including all potential predictors and moderators assessed before the start of treatment, as well as all outcome variables assessed during and after treatment, both in the STPP condition as well as in any comparison condition included in the study. The author will anonymise the data, so that the dataset is transferred without containing personal information that can lead back to individuals. Data can be submitted to the project in any format, and will then be converted to Stata.

\section{Data integrity check}

After the dataset has been transferred, the file will be checked to examine whether the data received match the data reported in the publication. For all treatment conditions included in the study, sample size, number of females, mean age, observed mean pretreatment depression scores, observed mean post-treatment score for the primary depression outcome and the number of missing cases for the latter will be calculated from the dataset received and checked against the published article for this purpose. Discrepancies will be resolved with the authors.
In addition, the data will be checked for invalid, out-ofrange, or inconsistent items. Furthermore, we will check the integrity of the randomisation for randomised studies by inspecting the balance of the potential predictor/ moderator variables across treatment arms.

For each study, we will list all predictor/moderator variables that were assessed, as well as all outcome variables, intermediate and follow-up assessments. We will also extract multiple STPP characteristics (eg, number of sessions, treatment format, STPP mode) and study design characteristics (eg, therapist training, treatment integrity check, use of a treatment manual; for a complete overview see $\left.^{4}\right)$. Finally, we will extract study validity criteria according to the Cochrane risk of bias assessment tool. ${ }^{17}$

After checking the data, the datasets will be standardised. For this purpose, a copy of each trial's raw data file will be recoded into a data file that matches the IPD meta-analysis database in terms of variables. Next, the individual study data files will be concatenated in one database structured by study and individual participant ID. After all data files have been recoded and entered, the data for each study will be checked with the original data file received for accuracy. A codebook document will be made that includes the coding of the individual studies as well as the coding of the combined study database. Coding for the database will be finalised when all data have been received from the study authors.

\section{Measures}

The primary outcome for this study is treatment efficacy as assessed by a continuous depression outcome measure at post-treatment. We have chosen depressive symptom status as the main outcome for this study as we consider this to be the primary target of STPP for depression. We chose continuous symptom level as the primary outcome, because we expect that the increased variance relative to dichotomous outcomes might facilitate the search for predictors and moderators. We have chosen post-treatment assessment as the primary end point as it is difficult to control additional treatment in the follow-up period in psychotherapy efficacy studies (eg, reference 18).

For each trial, we will identify the primary continuous depression outcome as defined by the study authors. All instruments explicitly measuring depression qualify in this regard. Different depression measures have probably been used and, therefore, we will standardise the depression outcomes by converting the depression scores into z-scores. Sensitivity analyses will be conducted using unstandardised scores for each depression measure that is assessed in the majority of studies included in the meta-analysis (eg, Hamilton Depression Rating Scale, ${ }^{19}$ Beck Depression Inventory ${ }^{20}$ ).

The secondary outcomes for this study are dichotomous depression outcomes for (1) response (a 50\% reduction in symptoms from pretreatment to post-treatment) and (2) remission (maximum absolute post-treatment scores reflecting normalisation). In addition, outcome measures other than depression will be collected (eg, anxiety 
symptoms, quality of life, interpersonal functioning). These can be considered tertiary outcomes.

Potential predictors and moderators include sociodemographic variables (eg, gender, age, education level, marital status, employment status, ethnicity), clinical variables (eg, number of previous depressive episodes, previous exposure to treatment, comorbid Axis I and II psychopathology, global assessment of functioning) and psychological patient characteristics (eg, personality organisation, attachment, interpersonal styles, childhood maltreatment, alexithymia). These are likely assessed differently in individual studies and will be standardised as well, for instance by converting scores into z-scores for continuous variables or by recoding variables into similar categories for categorical variables. In the latter case, we will consult study authors to confirm correctness of the recoding.

\section{Missing data}

All datasets received that contain IPD, at least one relevant outcome measure and at least one predictor/moderator variable will be considered for quantitative synthesis. We will examine the possibility of complete-case analyses by evaluating the extent of missing data as well as the possible reasons for missing data, and by comparing patients with complete data to patients with missing data. We will also assess whether the missing data can be considered missing at random. ${ }^{21}$ If complete-case analysis is not justifiable and data can be considered missing at random, we will impute missing data using multiple imputation, which is currently considered to be the most sophisticated method for handling missing data. ${ }^{22}$

We will generate one imputed dataset for each percent of missing data (eg, when $30 \%$ of the data are missing, we will create 30 imputed datasets). We will impute missing data by means of hierarchical imputation with fully conditional specification for the generalised linear model (FCS-GLM) ${ }^{23}$ which allows for preserving the heterogeneity across studies, non-normal distributions of variables and imputing systematically missing variables. FCS-GLM has shown to be a reliable procedure with advantageous properties for IPD meta-analyses with limited numbers of studies or studies with small sample sizes. ${ }^{24}$ FCS-GLM will be conducted in $R$. To ensure congeniality, imputation will be based on all variables that will be included in the meta-analysis model including their interactions as well as any variables that were identified to be predictive of missing values. ${ }^{24}$ If multiple imputation is pursued, we will conduct sensitivity analyses restricted to complete cases and compare the results. We will not pursue efforts to combine IPD with aggregate data from studies for which no IPD is available ${ }^{25}$ since the requisite treatment-covariate interactions are seldom reported in publications.

\section{Data analysis}

We will conduct IPD meta-analyses according to the one-stage approach, because that accounts for the correlation among model parameters when modelling interactions, offers the highest degree of flexibility for making the necessary assumptions when detecting treatment-covariate interactions, ${ }^{26}$ and provides a more exact likelihood in the case of small studies. ${ }^{27}$ We will conduct IPD meta-analyses using mixed models with restricted maximum likelihood to estimate between-study heterogeneity, which is recommended when there are few studies in the meta-analysis or studies have small sample sizes. ${ }^{28}$ Analyses will be conducted in Stata 'mixed'. We will apply the Kenward-Roger denominator-degrees-of-freedom adjustment for CIs, ${ }^{29}$ because it more adequately accounts for uncertainty than the standard Dersimonian-Laird estimator when the number of studies is small or when heterogeneity is present. ${ }^{30}$ To account for clustering of patients within studies and to preserve randomisation, we will include a random intercept for study and a random slope for baseline depression. We assume that these random effects will be normally distributed and this assumption will be assessed. We will assess heterogeneity by examining the SD of the predictor or moderator parameter estimates.

Separate analyses will be conducted for the identification of predictors and the identification of moderators of STPP efficacy. With regard to predictors, IPD from STPP conditions across all studies will be combined, regardless of study type (randomised controlled study, non-random comparative study, naturalistic study). For each of the potential predictors, a model will be estimated with baseline depression and the predictor's main effect. A random slope will be added to the predictor to examine if this results in a model improvement. If so, this predictor variable will be included with a random slope in subsequent analyses. Next, all predictors will be modelled simultaneously. Backward selection based on $\mathrm{P}$ value will be conducted until a final prediction model is obtained consisting of statistical significant predictors only. This prediction model will be validated in a held-out sample of the data.

With regard to moderators, analyses will be conducted separately for each comparison (eg, STPP vs control conditions, STPP vs other psychotherapy and STPP vs antidepressant medication). These analyses will only include IPD from randomised studies. For each of the potential moderators, a model will be estimated with baseline depression as well as the moderator's main effect and interaction with treatment. Next, to examine ecological bias, within-study and across-study interaction effects will be separated. ${ }^{25}$ If within-study and across-study interactions differ, ecological bias may be at play, and only the within-trial interaction will be interpreted in subsequent analyses. Finally, all significant treatment-covariate interactions, their main effects and higher order interactions will be modelled simultaneously. The resulting prediction model will be validated in a held-out sample of the data.

A number of sensitivity analyses will be conducted to examine the robustness of the findings. To examine the impact of study quality, we will conduct sensitivity analyses including only studies that score high on all quality 
criteria. In addition, we will conduct analyses in which we add the risk of bias items as covariates to the mixed-effects models, centred at a value indicating freedom of bias. ${ }^{31}$ We will also conduct sensitivity analyses to control for the effects of additional non-study treatment during the trial and in the follow-up period by adding collected data in this regard as covariates to the mixed-effects models. Finally, we will examine the impact of STPP characteristics (eg, STPP type, delivery mode) and study design characteristics (eg, therapist training, use of a treatment manual) by adding these variables to the mixed-effects models too.

Furthermore, to examine possible data availability bias, t-tests and $\mathrm{X}^{2}$ analyses will be conducted comparing the included studies with studies for which no participant-level data was obtained with regard to the extracted study characteristics. Conventional meta-analysis techniques will be used to examine differences in effect sizes between studies that contributed data and studies that did not. We will assess potential publication bias by assessing asymmetry in contour-enhanced funnel plots for meta-analyses including 10 or more trials, as is recommended by Sterne et al. ${ }^{32}$ The confidence in the cumulative body of evidence will be assessed according to the Grading of Recommendations Assessment, Development and Evaluation (GRADE) system. ${ }^{33}$

In addition, we will conduct explorative analyses using tree-based statistical learning. More specifically, we will use the generalised linear mixed-effects regression trees algorithm (glmertree ${ }^{34}$ ) in $\mathrm{R}$ to detect predictors and moderators of continuous and binary treatment outcomes. These analyses offer several advantages over more traditional mixed-effects modelling approaches, as they allow for the detection of non-linear and higher level interactions, allow for specifying a large number of potential predictor and moderator variables and involve less stringent assumptions about the distribution of the data. Furthermore, the result of applying glmertree consists of a decision tree that graphically depicts the effects of the relevant predictor and moderator variables. Such a tree can easily be interpreted, and applied in clinical decision-making. However, it should be noted that such tree-based analyses are exploratory in nature. We will assess the expected predictive accuracy on new data using $k$-cross validation ${ }^{35}$ and the stability of the resulting decision trees using subsampling methods. ${ }^{36}$ The glmertree analyses will be performed using observed, non-imputed data only, as it is currently unclear how to best specify the model for generating imputed data in tree-based analyses for clustered data.

\section{ETHICS AND DISSEMINATION}

Institutional review board (IRB) approval was not required for this project. IRB approval may be required for the investigators to share their primary data depending on their institution's policies. It is the responsibility of the investigators to obtain IRB approval if their institution's policies require them to do so. By signing the data-sharing agreement, the authors who share their data declare that those data were collected and transferred to our research group according to all applicable local and international laws and regulations, including but not limited to local IRB approval.

We intend to submit reports of the outcomes of this study for publication to international peer-reviewed journals in the fields of psychiatry or clinical psychology. We also intend to present the findings of this study at international scientific conferences aimed at psychotherapy researchers and clinicians.

\section{DISCUSSION}

We described the study protocol of a systematic review and meta-analysis of IPD to examine predictors and moderators of STPP efficacy for depression. The goal of this study is to collect the participant-level data of studies examining the efficacy of STPP for depression identified by a thorough literature search, and to combine these datasets in order to conduct IPD meta-analyses. The proposed study design allows for the examination of predictors and moderators of STPP efficacy with increased statistical power and can thus help to show which subsets of depressed patients specifically benefit from therapies based on psychoanalytic principles.

\section{Clinical and scientific relevance}

In contrast to how commonly STPPs are used in clinical practice, they are under-represented in current treatment guidelines for depressive disorders. Further high-quality research that extends and disseminates knowledge about the effectiveness of STPP is therefore called for. Research showing whether subsets of depressed patients can specifically benefit from this therapy modality, especially when compared with other treatments, is particularly needed. Knowledge of such moderators can have important implications for clinical practice, as it can be used to guide treatment selection and increase the efficiency of treatments for depression by helping patients and clinicians to make an informed choice concerning the best treatment option for a given patient.

Little is known about patient characteristics associated with STPP efficacy, because clinical trials often have relatively small sample sizes and limited statistical power to examine predictors and moderators of change. Moreover, prediction and moderation analyses in conventional meta-analyses are often based on study-level aggregates and are, therefore, prone to ecological bias. By means of IPD meta-analyses, these critical limitations can be overcome. IPD meta-analyses have been utilised more frequently in the medical field, but are newer in the field of psychiatry and clinical psychology. ${ }^{37}$ Although IPD meta-analyses have now been used to examine single moderators of CBT and pharmacotherapy efficacy for depression, ${ }^{20}{ }^{21}$ to date no IPD meta-analysis has been conducted concerning STPP. 


\section{Strengths and limitations}

IPD meta-analysis has several advantages over conventional meta-analysis, including increased statistical power to examine predictors and moderators. ${ }^{11}$ In addition, by collecting the primary data, access to predictor or moderator variables that might not have been reported in the published articles is gained; increasing the chance that aggregation of these variables across studies is possible. Other advantages of IPD meta-analysis over conventional meta-analysis include the possibility to (1) account for missing data at the individual participant level, so that, for instance, intention-to-treat analyses can be conducted, even though the original study reported completersonly analyses, (2) use the same statistical methods for imputing missing data and for conducting statistical analyses, thereby facilitating standardisation across studies, (3) standardise outcomes across studies, for instance by using equal cut-off points on a depression outcome measure when the primary studies used different cut-offs and (4) verify the results presented in the original studies, also by means of more sophisticated statistical techniques that were not available at time of publication in the case of older studies. ${ }^{38}$

IPD meta-analyses involve collection of original data from all the relevant trials worldwide that can be accessed. These data need to be prepared and checked before being included in the meta-analysis, and complex decisions on the data sometimes need to be made in order to ensure the accuracy of the outcomes. Therefore, it takes more time and resources to conduct an IPD meta-analysis than to conduct a conventional meta-analysis based solely on results extracted from published trial reports. ${ }^{38}$ However, the IPD approach can improve the quality of both the data and the analyses, and so the reliability of the results. Therefore, it is considered the 'gold standard' of meta-analysis. ${ }^{12}$

IPD meta-analyses also have a number of limitations. First, although IPD meta-analyses are generally considered the most reliable approach to evidence synthesis, ${ }^{12}$ this does not mean that they are bias-free and selection bias, publication bias and data availability bias need to be considered. ${ }^{39}$ We try to overcome this limitation by performing a systematic literature search that also aims to identify grey literature and unpublished work, and by testing for differences between studies for which IPD was and was not obtained. Second, IPD meta-analyses rely on variables previously assessed in individual studies and available across multiple trials. For this reason, it is possible that not all variables of interest can be examined as potential predictors or moderators. Third, and related, it is necessary to standardise predictor/moderator variables for the analyses, but doing so might involve recoding and possibly omitting important information for some variables. Fourth, although combining multiple trials reduces the possibility that predictors/moderators identified are chance findings in single-study samples, generalisability of the findings might still be limited to patients who volunteer to participate in scientific outcome research. Fifth, the predictors and moderators identified in this study can either apply specifically to STPP or can be more general factors associated with depression treatment efficacy. We intend to address this distinction in this study's outcome reports in the context of the predictors/ moderators identified.

\section{Conclusion}

We described the study protocol for a systematic review and meta-analysis of IPD aimed at examining if certain subgroups of depressed patients can benefit specifically from STPP. We will collect the participant-level data of studies examining the efficacy of STPP for depression identified by a thorough literature search. We will combine these datasets and conduct IPD meta-analyses to identify predictors and moderators of STPP efficacy. Knowledge of such predictors and moderators can have important implications for clinical practice, as they can, respectively, inform expectations of treatment efficacy for individual patients, and help patients and clinicians to make an informed choice concerning the best treatment option for a given patient.

\section{Author affiliations}

${ }^{1}$ Department of Clinical, Neuro and Developmental Psychology, Amsterdam Public Health research institute, Vrije Universiteit Amsterdam, Amsterdam, Netherlands ${ }^{2}$ Centre for Emotions \& Health, Dalhousie University, Halifax, Nova Scotia, Canada ${ }^{3}$ Gordon F. Derner School of Psychology, Adelphi University, Garden City, New York, USA

${ }^{4}$ Department of Psychiatry, University of Pennsylvania, Philadelphia, Pennsylvania, USA

${ }^{5}$ Department of Research, Arkin Mental Health Care, Amsterdam, Netherlands ${ }^{6}$ Department of Methodology and Statistics, Leiden University, Leiden, Netherlands ${ }^{7}$ Department of Clinical, Educational and Health Psychology, University College London, London, UK

${ }^{8}$ Department of Psychology, Vanderbilt University, Nashville, Tennessee, USA ${ }^{9}$ University Library, Vrije Universiteit Amsterdam, Amsterdam, Netherlands

${ }^{10}$ Dutch Psychoanalytic Institute, Arkin Mental Health Care, Amsterdam, Netherlands

${ }^{11}$ Department of Health Sciences, Vrije Universiteit Amsterdam, Amsterdam,

Netherlands

${ }^{12}$ Department of Epidemiology and Biostatistics, VU University Medical Center Amsterdam, Amsterdam, Netherlands

Contributors ED, AAA, JPB, JJMD, MF, SDH, EPJ, SCMdM, JMT, JWRT, HLV, EW and PC made substantial contributions to the study design. ED, AAA, JPB, MBCG, JJMD, PF, SDH, JMT, HLV and PC made substantial contributions to the acquisition of individual participant data. ED, EW and PC drafted the manuscript. ED, AAA, JPB, MBCG, JJMD, MF, PF, SDH, EPJ, SCMdM, JMT, JWRT, HLV, EW and PC revised it critically for important intellectual content and approved the final version of this manuscript. ED is the guarantor of the review.

Funding An American Psychoanalytic Association Research Fund supported this work.

Disclaimer The funder had no role in the development of this study protocol, nor was there editorial direction or censorship from the sponsor in this manuscript.

Competing interests AAA practices and provides training in methods of STPP and receives royalties from a book he wrote on STPP. JPB has given talks on STPP at workshops and conferences for which organisers often have not paid for travel and accommodations. JPB and JJMD receive royalties from books on STPP they have coauthored. JMT has given talks on STPP at workshops and conferences for which organisers have paid for travel and accommodations. PF declares being Chief Executive of the Anna Freud Centre (UK). He teaches mentalisation-based treatment trainings and dynamic-interpersonal therapy trainings in the UK and internationally. He is the Co-PI on The IMPACT Study - The Effectiveness of Psychological Treatment for Depressed Adolescents, and Consultant to the Child and Family 
Program at the Menninger Department of Psychiatry and Behavioural Sciences at Baylor College of Medicine, Houston, Texas, USA. PF also receives royalties from books on interpersonal psychotherapy which he has coauthored with others. HLV and SCMdM are trainers and registered supervisors of short-term psychodynamic supportive psychotherapy. They also receive royalties from books on STPP they have coauthored. SDH reports no financial conflicts, but acknowledges an intellectual passion for the cognitive and behavioural interventions for depression. ED, MBCG, MF, JWRT, EW and PC declare that they have no known conflicts of interest

Patient consent Not required.

Provenance and peer review Not commissioned; externally peer reviewed.

Open Access This is an Open Access article distributed in accordance with the Creative Commons Attribution Non Commercial (CC BY-NC 4.0) license, which permits others to distribute, remix, adapt, build upon this work non-commercially, and license their derivative works on different terms, provided the original work is properly cited and the use is non-commercial. See: http://creativecommons.org/ licenses/by-nc/4.0/

(c) Article author(s) (or their employer(s) unless otherwise stated in the text of the article) 2018. All rights reserved. No commercial use is permitted unless otherwise expressly granted.

\section{REFERENCES}

1. Kessler RC. The costs of depression. Psychiatr Clin North Am 2012:35:1-14.

2. World Health Organization. Depression and other common mental disorders. Global health estimates. http://apps.who.int/iris/bitstream/ 10665/254610/1/WHO-MSD-MER-2017.2-eng.pdf?ua=1 (accessed July 2017).

3. Marcus SC, Olfson M. National trends in the treatment for depression from 1998 to 2007. Arch Gen Psychiatry 2010;67:1265-73.

4. Driessen E, Hegelmaier LM, Abbass AA, et al. The efficacy of shortterm psychodynamic psychotherapy for depression: A meta-analysis update. Clin Psychol Rev 2015;42:1-15.

5. Barber JP. Toward a working through of some core conflicts in psychotherapy research. Psychother Res 2009;19:1-12.

6. Kraemer HC, Wilson GT, Fairburn CG, et al. Mediators and moderators of treatment effects in randomized clinical trials. Arch Gen Psychiatry 2002;59:877-83.

7. Driessen E, Cuijpers P, Hollon SD, et al. Does pretreatment severity moderate the efficacy of psychological treatment of adult outpatient depression? A meta-analysis. J Consult Clin Psychol 2010;78:668-80.

8. Driessen E, Smits N, Dekker JJ, et al. Differential efficacy of cognitive behavioral therapy and psychodynamic therapy for major depression: a study of prescriptive factors. Psychol Med 2016;46:731-44.

9. Barber JP, Barrett MS, Gallop R, et al. Short-term dynamic Psychotherapy Versus Pharmacotherapy for major depressive disorder. J Clin Psychiatry 2012;73:66-73.

10. Fisher DJ, Carpenter JR, Morris TP, et al. Meta-analytical methods to identify who benefits most from treatments: daft, deluded, or deft approach? BMJ 2017;356:j573.

11. Lambert PC, Sutton AJ, Abrams KR, et al. A comparison of summary patient-level covariates in meta-regression with individual patient data meta-analysis. J Clin Epidemiol 2002;55:86-94.

12. Stewart LA, Parmar MK. Meta-analysis of the literature or of individual patient data: is there a difference? Lancet 1993;341:418-22.

13. Driessen E, Cuijpers $P$, de Maat SC, et al. The efficacy of short-term psychodynamic psychotherapy for depression: a meta-analysis. Clin Psychol Rev 2010;30:25-36.

14. Cuijpers $P$, van Straten A, Warmerdam L, et al. Psychological treatment of depression: a meta-analytic database of randomized studies. BMC Psychiatry 2008;8:36.

15. Cuijpers P, Weitz E, Twisk J, et al. Gender as predictor and moderator of outcome in cognitive behavior therapy and pharmacotherapy for adult depression: an "individual patient data" meta-analysis. Depress Anxiety 2014;31:941-51

16. Weitz ES, Hollon SD, Twisk J, et al. Baseline depression severity as moderator of depression outcomes between cognitive behavioral therapy vs pharmacotherapy: an individual patient data metaanalysis. JAMA Psychiatry 2015;72:1102-9.

17. Higgins JPT, Green S. Cochrane handbook for systematic reviews of interventions version 5.1.0 [updated March 2011]: The Cochrane Collaboration. http://www.cochrane-handbook.org

18. Driessen E, Van HL, Don FJ, et al. The efficacy of cognitivebehavioral therapy and psychodynamic therapy in the outpatient treatment of major depression: a randomized clinical trial. Am J Psychiatry 2013;170:1041-50.

19. Hamilton M. A rating scale for depression. J Neurol Neurosurg Psychiatry 1960;23:56-62.

20. Beck AT, Ward $\mathrm{CH}$, Mendelson $\mathrm{M}$, et al. An inventory for measuring depression. Arch Gen Psychiatry 1961;4:561-71.

21. Sterne JA, White IR, Carlin JB, et al. Multiple imputation for missing data in epidemiological and clinical research: potential and pitfalls. BMJ 2009;338:b2393.

22. Donders AR, van der Heijden GJ, Stijnen T, et al. Review: a gentle introduction to imputation of missing values. J Clin Epidemiol 2006:59:1087-91.

23. Jolani S, Debray TP, Koffijberg H, et al. Imputation of systematically missing predictors in an individual participant data meta-analysis: a generalized approach using MICE. Stat Med 2015;34:1841-63.

24. Audigier V, White IR, Jolani S, et al. Multiple imputation for multiplevel data with continuous and binary variables. https://arxiv.org/abs/1702 00971 (accessed July 2017).

25. Riley RD, Lambert PC, Staessen JA, et al. Meta-analysis of continuous outcomes combining individual patient data and aggregate data. Stat Med 2008;27:1870-93.

26. Debray TP, Moons KG, van Valkenhoef $\mathrm{G}$, et al. Get real in individual participant data (IPD) meta-analysis: a review of the methodology. Res Synth Methods 2015;6:293-309.

27. Burke DL, Ensor J, Riley RD. Meta-analysis using individual participant data: one-stage and two-stage approaches, and why they may differ. Stat Med 2017;36:855-75.

28. Higgins JP, Whitehead A, Turner RM, et al. Meta-analysis of continuous outcome data from individual patients. Stat Med 2001;20:2219-41.

29. Kenward MG, Roger JH. Small sample inference for fixed effects from restricted maximum likelihood. Biometrics 1997;53:983-97.

30. Cornell JE, Mulrow CD, Localio R, et al. Random-effects metaanalysis of inconsistent effects: a time for change. Ann Intern Med 2014;160:267-70.

31. Higgins JPT, Thompson SG, Spiegelhalter DJ. A re-evaluation of random-effects meta-analysis. J $R$ Stat Soc Ser A Stat Soc 2009;172:137-59.

32. Sterne JA, Sutton AJ, loannidis JP, et al. Recommendations for examining and interpreting funnel plot asymmetry in meta-analyses of randomised controlled trials. BMJ 2011;343:d4002.

33. Guyatt $\mathrm{GH}$, Oxman AD, Vist GE, et al. GRADE: an emerging consensus on rating quality of evidence and strength of recommendations. BMJ 2008;336:924-6.

34. Fokkema M, Smits N, Zeileis A, et al. Detecting treatment-subgroup interactions in clustered data with generalized linear mixed-effects model trees. 2015. Working Papers in Economics and Statistics, No. 2015-10.

35. Friedman J, Hastie T, Tibshirani R. The Elements of Statistical Learning. 2nd edn. Berlin: Springer, 2009.

36. Philipp M, Zeileis A, Strobl C. A toolkit for stability assessment of tree-based learners. 2016. Working Papers in Economics and Statistics No. 2016-11.

37. Simmonds M, Stewart G, Stewart L. A decade of individual participant data meta-analyses: A review of current practice. Contemp Clin Trials 2015;45:76-83.

38. Riley RD, Lambert PC, Abo-Zaid G. Meta-analysis of individual participant data: rationale, conduct, and reporting. BMJ 2010;340:c221.

39. Ahmed I, Sutton AJ, Riley RD. Assessment of publication bias, selection bias, and unavailable data in meta-analyses using individual participant data: a database survey. BMJ 2012;344:d7762. 